\title{
Expression and clinical significance of ubiquitin-specific-processing protease 34 in diffuse large B-cell lymphoma
}

\author{
CHUNYAO LI $^{1 *}$, LANSHAN HUANG ${ }^{1}{ }^{*}$, HUIPING LU $^{1}$, WEI WANG ${ }^{2}$, GANG CHEN $^{1}$, \\ YONGYAO GU ${ }^{1}$, QIANPING ZHOU ${ }^{1}$, ZHIGANG PENG ${ }^{3}$ and ZHENBO FENG ${ }^{1}$ \\ ${ }^{1}$ Department of Pathology, The First Affiliated Hospital of Guangxi Medical University, \\ Nanning, Guangxi Zhuang Autonomous Region 530021, P.R. China; ${ }^{2}$ Department of Hematopathology, \\ The University of Texas MD Anderson Cancer Center, Houston, TX 77030, USA; \\ ${ }^{3}$ Department of Medical Oncology, The First Affiliated Hospital of Guangxi Medical University, \\ Nanning, Guangxi Zhuang Autonomous Region 530021, P.R. China
}

Received March 11, 2017; Accepted October 13, 2017

DOI: $10.3892 / \mathrm{mmr} .2018 .9447$

\begin{abstract}
Ubiquitin-specific-processing protease 34 (USP34) is a deubiquitinase that is involved in the pathogenesis of various cancers. Its roles in diffuse large B-cell lymphoma (DLBCL) are unknown. The present study aimed to determine the level of USP34 expression and to explore its association with clinicopathological features and prognosis in patients with DLBCL; a total of 30 cases of reactive lymphoid hyperplasia and 131 cases of DLBCL were included in this study. The level of USP34 expression was examined by immunohistochemistry (IHC), and correlations between USP34 expression and clinicopathological features and prognosis were analyzed. In addition, mutations, expression and clinical significance of USP34 in DLBCL were evaluated using data from The Cancer Genome Atlas (TCGA). USP34 expression was significantly higher in DLBCL compared with expression in reactive lymphoid hyperplasia. In DLBCL, overexpression of USP34 was associated with older age, germinal center B cell-like
\end{abstract}

Correspondence to: Professor Zhigang Peng, Department of Medical Oncology, The First Affiliated Hospital of Guangxi Medical University, 6 Shuangyong Road, Nanning, Guangxi Zhuang Autonomous Region 530021, P.R. China

E-mail: drpzg001@163.com

Professor Zhenbo Feng, Department of Pathology, The First Affiliated Hospital of Guangxi Medical University, 6 Shuangyong Road, Nanning, Guangxi Zhuang Autonomous Region 530021, P.R. China

E-mail: guanghu1963@126.com

*Contributed equally

Key words: diffuse large B-cell lymphoma, ubiquitinspecific-processing protease 34, clinicopathological significance, expression, prognosis
(GCB) subtype, multiple extranodal involvements and higher International Prognostic Index (IPI) scores. No significant association was identified between USP34 protein level and patient survival. In the TCGA dataset, low USP34 mRNA expression was demonstrated to be associated with a poor disease-free survival (DFS), but not with overall survival (OS) in patients with DLBCL. In conclusion, high expression of USP34 protein in DLBCL was associated with older age, GCB subtype, multiple extranodal involvement and high IPI scores of DLBCL. USP34 may be a valuable marker for the assessment of patients with DLBCL, and further studies are needed to clarify USP34 expression on DLBCLs.

\section{Introduction}

Current cancer statistics indicate that non-Hodgkin lymphoma (NHL) has the sixth highest morbidity rate and the ninth highest mortality rate of all cancers (1). Diffuse large B cell lymphoma (DLBCL) is the most common type of NHL, and is an aggressive lymphoma with heterogeneous morphology, various molecular abnormalities and different clinical outcomes $(2,3)$. DLBCL has been stratified based on gene expression profiling and is classified into two subgroups: Germinal center B cell-like (GCB) and non-germinal center B cell-like (non-GCB). This demonstrates the different responses to treatments and clinical outcomes (2); however, a recent study has reported that there was no difference in survival rate between these two groups of treatment (4). The standard treatment for patients with DLBCL is rituximab monoclonal antibody combined with cyclophosphamide, doxorubicin, vincristine and prednisone (R-CHOP) chemotherapy, which has significantly improved the outcome (3). Although the 5-year overall survival (OS) and the event-free survival of patients with DLBCL have increased to 70 and $52 \%$, respectively, one-third of patients experience refractory disease or relapse following treatment $(5,6)$. Therefore, studies investigating novel signaling pathways and potential therapeutic targets for DLBCL are necessary. 
The ubiquitin proteasome system is vital for protein degradation and maintains normal cellular biological processes, including cell cycle, apoptosis, differentiation, DNA repair and signaling pathways (7-9). Ubiquitination may be reversed by deubiquitinating enzymes (DUBs), which remove ubiquitin from ubiquitinated proteins. Deubuiquitination by DUBs is involved in the pathogenesis of breast cancer and inflammation (7). Ubiquitin-specific-processing protease (USPs), the most prominent family of DUBs, serve fundamental roles in the ubiquitin system (10), and the role of various USPs has been examined in cancer pathogenesis. For example, USP10 was reported to be an independent poor prognostic factor of gastric carcinoma (11). USP14 was revealed to induce cell proliferation and promote apoptosis in breast cancer and epithelial ovarian cancer cells $(7,12)$. In addition, previous studies have demonstrated that USP22 was a poor prognostic factor in oral squamous cell carcinoma and invasive breast cancer $(8,13)$.

USP34 is located on chromosome $2 \mathrm{p} 15$ and encodes a deubiquitinating enzyme that was previously demonstrated to stabilize $\beta$-catenin and modulate Wnt signaling (14). USP34 was also demonstrated to prevent constitutive activation of Toll-dependent immune signaling (15). In addition, USP34 was reported to negatively regulate nuclear factor $(\mathrm{NF})-\kappa \mathrm{B}$ signaling in $\mathrm{T}$ cell receptor-dependent lymphocyte activation (16). A number of recent studies have linked USP34 with various diseases. For example, USP34 may serve a potential role in the pathophysiology of polycystic ovary syndrome (PCOS), although no significant correlation has been identified between PCOS and USP34 gene polymorphisms in the Chinese women involved in that study (17). A previous case study of 2p15-p16.1 microdeletion syndrome revealed that USP34 may affect the fundamental developmental process of nervous system (18). In addition, a gain of USP34 due to duplication in the 2p15-p16.1 region was frequently observed in patients with DLBCL compared with controls by qPCR (19-21), and USP34 may participate in the transformation from follicular lymphoma to DLBCL (19). Recent studies using array comparative genomic hybridization, single-nucleotide polymorphism (SNP)-chips and gene expression profiling analyses have revealed high levels of USP34 expression in DLBCL tissue chips (18-22).

The present study aimed to examine the expression level of USP34 in DLBCL and to explore its association with the patient clinicopathological features and prognosis. In addition, a dataset from The Cancer Genome Atlas (TCGA) was used to assess genetic mutations, expression and clinical significance of USP34 in DLBCL.

\section{Materials and methods}

Patients and tissue samples. A total of 131 patients with DLBCL and 30 patients with reactive lymphoid hyperplasia were included in this study. Patients with DLBCL were recruited between July 1, 2011 and September 30, 2015, and all DLBCL diagnoses were confirmed following 2008 WHO criteria. The median age for patients with DLBCL at diagnosis was 54 years (range 18-86 years). The follow-up time ranged between 0.5 and 63 months. None of the patients had a history of immunodeficiency. The tissue used for initial diagnosis (as detailed below) was used to measure USP34 protein expression levels. For DLBCL, these tissues included lymph nodes $(n=43)$, mainly neck and inguinal lymph nodes, and extranodal lesions $(n=88)$, mainly in stomach, intestines, liver, spleen and brains. Of the 131 patients with DLBCL, 78 were treated with chemotherapy, among which 39 received R-CHOP treatment, 29 received CHOP therapy and 10 received other chemotherapy (methotrexate or temozolomide). Hematoxylin and eosin (H\&E) staining on these tissues was performed at room temperature in order to observe the morphology using a light microscope (Olympus BX53; Olympus Corporation, Tokyo, Japan). The H\&E staining dyeing procedure is as follows: i) The paraffin tissues were dewaxed with xylene scavenger for about $20 \mathrm{~min}$. ii) Different concentrations of ethanol $(95,80$ and $70 \%$ ethanol) were used to remove xylene. iii) distilled water was used for $1 \mathrm{~min}$ to remove alcohol. iv) Subsequently, tissues were immersed in hematoxylin dye for $1 \mathrm{~min}$, and v) washed with tap water for 1 min. vi) Hydrochloric acid $(0.5 \%)$ was used to differentiate alcohol $2 \mathrm{sec}$ and tap water for $2 \mathrm{sec}$. vii) Subsequently, tissues were immersed in $1 \%$ lithium carbonate solution for $1 \mathrm{~min}$, washed in tap water for $1 \mathrm{~min}$, distilled water for $1 \mathrm{~min}$, and immersed in $0.5 \%$ eosin for $10 \mathrm{sec}$. viii) Subsequently, tissues were washed with distilled water for $2 \mathrm{sec}$, dehydrated with different concentrations of alcohol, and ix) xylene was used as a clearing reagent to remove the alcohol from the tissues. Finally, tissues were sealed with neutral balsam. Furthermore, clinical stage was assessed based on WHO Ann Arbor Criteria (2008), and patient status was evaluated with Eastern Cooperative Oncology Group (ECOG) and International Prognostic Index (IPI) scores. Additionally, 131 patients with DLBCL were utilized to analyze the corresponding clinical information. This study is approved by Ethics Committee of the First Affiliated Hospital of Guangxi Medical University, and written informed consent was obtained from each patient prior to enrollment in the study.

Immunohistochemistry. Formalin-fixed paraffin-embedded tissues were sectioned $(4 \mu \mathrm{m})$ and used for immunohistochemical analysis. The slides were baked at $65^{\circ} \mathrm{C}$ for $6 \mathrm{~h}$, and subsequently dewaxed (23), the slides were rehydrated through an ethanol series. EDTA buffer PH:9.0 solution (1:50; Zhongshan Jinqiao Biotechnology Co., Ltd., Beijing, China) for antigen retrieval following $3 \mathrm{~min}$ of high-pressure, and the endogenous peroxidase activity was inhibited with $3 \%$ hydrogen peroxide for $20 \mathrm{~min}$ at room temperature. The primary antibody against USP34 (1:200; catalog no. ab91617; Abcam, Cambridge, UK) was added and incubated at $4^{\circ} \mathrm{C}$ overnight. Tissue sections were also incubated with primary antibodies against B-cell lymphoma (BCL)-2, BCL6 (Fuzhou Maixin Biotech Co., Ltd., Ningde, China), CD20, mutated melanoma-associated antigen (MUM)-1, CD10 and Ki67 (Ready to use; OriGene Technologies, Inc., Beijing, China) were incubated at $37^{\circ} \mathrm{C}$ for $1 \mathrm{~h}$. Sections were subsequently incubated with secondary antibodies (Polymerized HRP-Anti Mouse/Rabbit IgG; Shanghai Changdao Biotechnology Co., Ltd., Shanghai, China) for $30 \mathrm{~min}$ at $37^{\circ} \mathrm{C}$. Following incubation, secondary antibodies were removed and slides were washed three times with PBS. Expression was developed with 3,3'-diaminobenzidine buffer, and slides were counterstained with hematoxylin. Following staining, sections were 

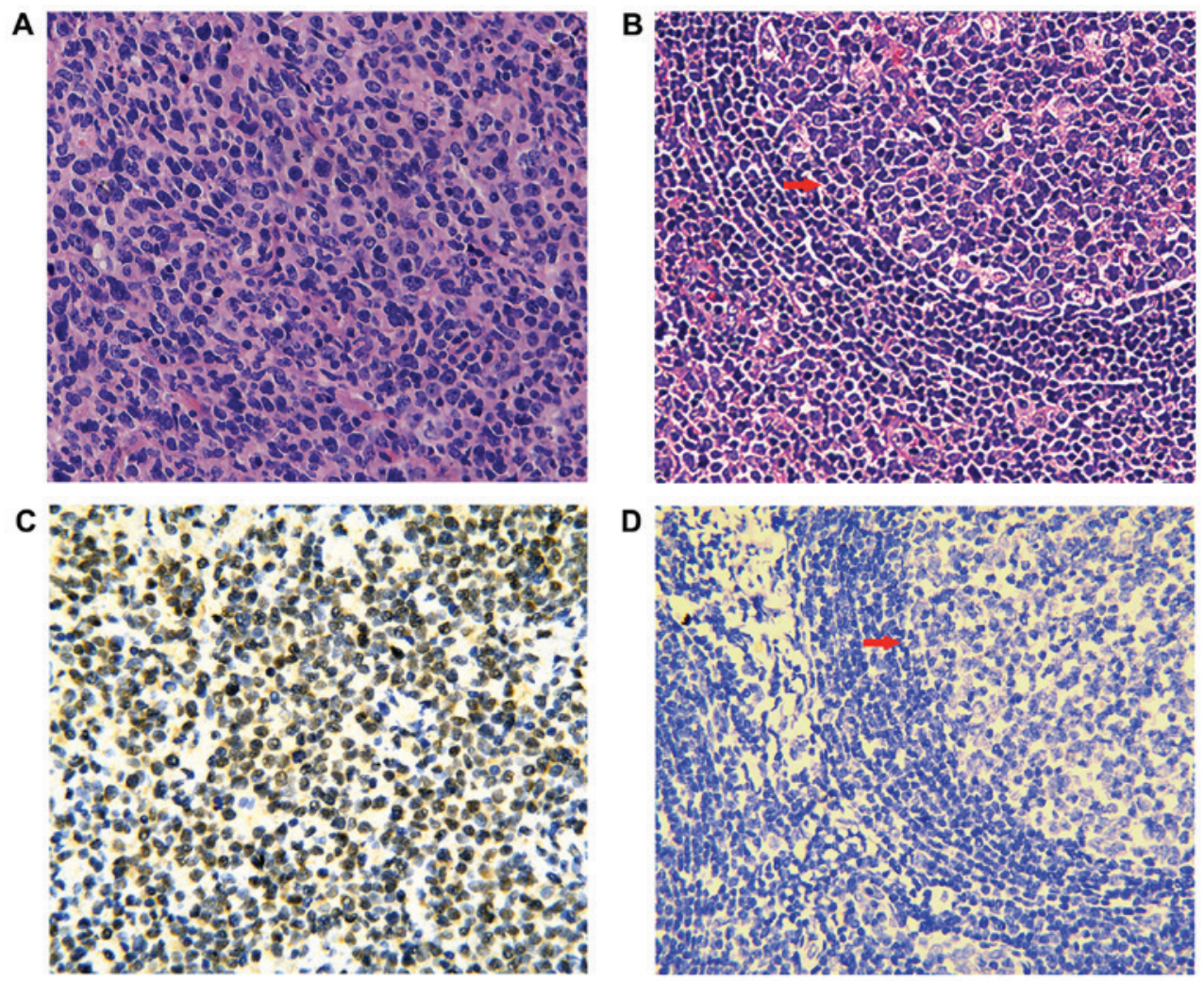

Figure 1. Morphology and IHC results of USP34 expression in DLBCL from lymph nodes and reactive hyperplasia of lymph node tissues. (A) DLBCL tissue, H\&-E staining; magnification, $x$ 400. (B) Reactive hyperplasia of lymph node tissue, H\&E staining; magnification, x400. (C) IHC of USP34 in DLBCL tissue; magnification, x400. (D) IHC of USP34 in reactive hyperplasia of lymph node tissue; magnification, x400; DLBCL, diffuse large B-cell lymphoma; H\&E, hematoxylin and eosin; IHC, immunohistochemistry. Arrows indicate follicular hyperplasia.

dehydrated for transparency and slides were sealed with neutral balsam. The positive control tissues were selected according to a previous study (23) and primary antibodies were replaced with PBS in the negative control groups.

Evaluation of immunohistochemical staining. From a previous study it was demonstrated that USP34 is expressed in the nucleus of DLBCL cells, and in the cytoplasm and/or nucleus of reactive lymphoid hyperplasia cells (19). As no previously published studies describe the detection of USP34 protein expression with immunohistochemical method in tumors, the immunohistochemical evaluation standards of the USP family, including USP2a (24), USP9X (25), USP10 (11), USP14 (7,12), USP22 (13,26-29), USP28 (30) and USP39 (31), in tumors were used, and the middle evaluation standard value was considered as the cut-off value of USP34. The expression level of USP34protein was scored with a combination of intensity and percentage of positive tumor cells $(26,27,31)$. The staining intensity of USP34 was scored as follows: 0, colorless; 1, light yellow; 2, yellowish brown; and 3, dark brown. The percentage of USP34-positive DLBCL cells was scored as follows: 0, $\leq 5 \%$; 1, 6-25\%; 2, 26-50\%; 3, 51-75\%; and 4, >75\%. Overall USP34 expression level was calculated by multiplying the percentage score with the intensity score, such that a score $<6$ was considered as low expression and a score $\geq 6$ was defined as high expression. BCL2, CD20 and CD10 were observed in cytomembrane of DLBCL cells; BCL6, MUM1 and Ki67 were stained in nucleus of DLBCL cells. The selection of antibodies and the evaluation standards $(32,33)$ were kept with clinical proposals applied by the Department of Pathology, The First Affiliated Hospital of Guangxi Medical University (Nanning, China) based on the current clinical practice. Tissue samples with $>25 \%$ stained tumor cells were considered as positive for BCL2, CD20, CD10, BCL6 and MUM1, and $>70 \%$ for Ki67. All slides were evaluated independently by two experienced pathologists.5 fields were selected using the Olympus BX53 and 1,000 cells were counted per field at high magnification (x400), repeated at least three times to evaluate the immunoreactivity.

cBioPortal analysis of TCGA dataset. Genetic alterations of USP34 in 48 DLBCL cases from TCGA (cancergenome.nih. gov/) $(34,35)$ were analyzed with 'OncoPrint' of cBioPortal (www.cbioportal.org) (36). The clinicopathological and survival significances of USP34 were displayed in the 'plot' and 'survival' modules of cBioPortal, respectively. The integral clinical data of USP34 mRNA expression in 48 DLBCL cases were downloaded from cBioPortal, and the correlations ofUSP34 and clinical features of DLBCL were analyzed by SPSS 22.0 (IBM Corp, Armonk, NY, USA), with $r>0$ indicating a positive correlation and $\mathrm{r}<0$ indicating a negative correlation. In addition, the network graph of USP34 and possible related pathogenic genes in DLBCL were downloaded from cBioPortal.

Statistical analysis. Statistical analysis was analyzed using SPSS 22.0 (IBM Corp., Armonk, NY, USA). Categorical data was calculated by $\chi^{2}$ test and Spearman's rank 
correlation, and data are presented as the mean \pm standard deviation. Kaplan-Meier analysis was applied to estimate OS, progression-free survival (PFS) and Disease-free survival (DFS), and the differences between curves were measured with log-rank test. Multivariate analysis of prognosis was assessed by Cox proportional hazards model. $\mathrm{P}<0.05$ was considered to indicate a statistically significant difference.

\section{Results}

Morphology and immunohistochemistry. H\&E stained sections of DLBCL and reactive lymphoid hyperplasia tissues were evaluated under a microscope. Morphologically, DLBCL is characterized with diffuse infiltration of tumor cells with the destruction of normal architecture in lymph nodes or extranodal tissues. Lymphoma cells had abundant cytoplasm, vesicular chromatin and inconspicuous to distinct nucleolus. The nucleus of tumor cells was equal to or larger than the nucleus of background macrophages (Fig. 1A). The main histologic changes of reactive lymphoid hyperplasia manifested follicular hyperplasia, paracortical lymphoid hyperplasia or sinus histiocytosis, while the normal structure maintained (Fig. 1B).

USP34 protein expression was primarily detected in the nucleus of DLBCL cells (Fig. 1C), whereas in lymph nodes with reactive hyperplasia, it was mainly located in cytoplasm and nucleus of cells in germinal center, medullary cord and medullary sinus (Fig. 1D). The number of USP34+ cells was significantly higher in DLBCL (nucleus; 58.8\%; 77/131) compared with those cells in reactive lymphoid hyperplasia tissue (cytoplasm and/or nucleus; 36.7\%; 11/30; $\mathrm{P}=0.028$ ).

Association of USP34 expression and clinical features in patients with DLBCL. Correlations between USP34 protein expression and clinicopathological parameters were assessed in 131 patients with DLBCL (Table I). The overexpression of USP34 was associated with older age $\left(\chi^{2}=4.979 ; \mathrm{P}=0.026\right), \mathrm{GCB}$ subtype $\left(\chi^{2}=3.871 ; \mathrm{P}=0.049\right)$, multiple extranodal involvements $\left(\chi^{2}=4.401 ; \mathrm{P}=0.036\right)$ and high IPI scores $\left(\chi^{2}=3.897 ; \mathrm{P}=0.048\right)$. By contrast, the expression of USP34 protein was not significantly correlated with sex, clinical stage, B symptoms (37) lactate dehydrogenase (LDH), hemoglobin (Hb), ECOG score, BCL2 expression status or Ki67 expression status ( $\mathrm{P}>0.05)$.

Survival analysis of patients with DLBCL. Of the 131 patients with DLBCL, the median follow-up time was 27.1 months (ranging between 0.5 and 63 months). The 3-year and 5-year OS rates were $54.9 \%$ [95\% confidence interval (CI); 49.8-60.0\%] and 29.4\% (95\% CI; 22.1-36.7\%), respectively. In univariate analysis, unfavorable clinical variables for prognosis were elderly age $(\mathrm{P}=0.036)$, advanced clinical stage III-IV $(\mathrm{P}=0.035)$, high LDH level $(\mathrm{P}<0.001)$, high IPI scores $(\mathrm{P}<0.001)$, non-GCB subtype $(\mathrm{P}=0.031)$ and positive BCL2 expression ( $\mathrm{P}=0.028$; Fig. 2A-F, respectively; Table II), whereas USP34 protein expression was not associated with OS or PFS (P>0.05; Fig. $2 \mathrm{G}$ and H, respectively). To eliminate the influence of non-chemotherapy on the prognosis, survival analysis of 78 patients who received chemotherapy were further analyzed. Patients with DLBCL that had clinical stage III-IV $(\mathrm{P}=0.021)$, high LDH $(\mathrm{P}<0.001)$, high IPI scores $(\mathrm{P}=0.001)$ or
non-GCB subtype ( $\mathrm{P}=0.044)$, had worse prognosis (Fig. 3A-D, respectively; Table III). In addition, no significant association was identified between the expression of USP34 and OS or PFS (P $>0.05$; Fig. 3E and F, respectively).

TCGA data analysis via cBioPortal platform. Of the 48 patients with DLBCL (TCGA, provisional) in cBioPortal, 15 (31\%) had genetic alterations of USP34 in 'OncoPrint', which included mRNA upregulation, in-frame and missense mutations (Fig. 4). To better understand the target genes associated with USP34 in NBCLB, online predictions from cBioPortal was used, and these genes (PSP27A, TNKS2, AXIN2, AXIN1, UBA52, TNKS, RNF146, ATM) were regarded as the potential target genes of USP34 and an interaction gene network of USP34 with other genes in DLBCL is provided in the Fig. 5. No significant correlations were identified between USP34 mRNA expression level and clinical stages (Fig. 6A). A total of 4 out of the 15 patients with alterations were deceased, and the median survival time was 116.72 months; and 5 out of the 32 cases without alterations were deceased, and the median survival time was 211.07 months. No significant association was demonstrated between USP34 genetic alterations with OS (Fig. 6B) in the present data, respectively, data also showed no significance with disease free survival and OS in TCGA dataset (DFS; P>0.05; Fig. 6C. OS; P>0.05; Fig. 6D). In TCGA dataset 47 cases of DLBCL contained adequate clinical information. The average expression level of USP34 mRNA was regarded as the cut-off value. No significant relationship was identified between USP34 mRNA level and clinical parameters $(\mathrm{P}>0.05)$ (data not shown). In univariate survival analysis, no clinical factor was found to be associated with OS ( $\mathrm{P}=0.994$; Fig. 7A). However, patients with low USP34 expression ( $\mathrm{P}=0.049$; Fig. 7B), stage III-IV $(\mathrm{P}=0.045)$, high ECOG score $(\mathrm{P}=0.007)$, advanced IPI scores $(\mathrm{P}=0.018)$ or non-chemotherapy $(\mathrm{P}=0.002)$ were indicated to be associated with poor DFS. Although USP34 mRNA level was associated with DFS, the OS was not affected.

\section{Discussion}

USP34 is a deubiquitinase that has become a topic of interest in recent years $(16,17)$. Previous studies have reported that USP34 may be involved in the pathogenesis of various diseases $(14,15)$; however, the role of USP34 in DLBCL remained to be determined. Thus, it is important to investigate whether USP34 serves a role in DLBCL pathogenesis. In the present study, the expression level and the clinical significance of USP34 in 131 patients with DLBCL were analyzed, and it was demonstrated that USP34 is expressed at higher levels in DLBCL compared with reactive lymphoid hyperplasia. In addition, increased of USP34 expression was associated with older age, GCB subtype, multiple extranodal site involvements and high IPI scores in patients with DLBCL $(3,6)$. In the present study, positive USP34 expression was more often to be detected in patients with GCB immunophenotype. Consistent with this finding, the increased expression of USP34 was detected by SNP-chip in GCB subtype of DLBCL cases $(20,21)$. A previous study on DLBCL reported a gain of $2 \mathrm{p} 15$ and $2 \mathrm{p} 16.1$ was more frequent in the GCB subgroup compared with the non-GCB 
Table I. Correlation of USP34 protein expression level and clinicopathological parameters of 131 DLBCL cases.

\begin{tabular}{|c|c|c|c|c|c|c|c|}
\hline \multirow[b]{2}{*}{ Clinicopathological parameters } & \multirow[b]{2}{*}{$\mathrm{n}$} & \multicolumn{2}{|c|}{ USP34 } & \multirow[b]{2}{*}{$\chi^{2}$} & \multirow[b]{2}{*}{ P-value } & \multirow[b]{2}{*}{$\mathrm{r}$} & \multirow[b]{2}{*}{ P-value } \\
\hline & & + & - & & & & \\
\hline \multicolumn{8}{|l|}{ Sex } \\
\hline Male & 77 & 48 & 29 & 0.084 & 0.772 & -0.025 & 0.774 \\
\hline Female & 54 & 35 & 19 & & & & \\
\hline \multicolumn{8}{|l|}{ Age } \\
\hline$>60$ & 49 & 37 & 12 & 4.979 & $0.026^{\mathrm{a}}$ & 0.195 & $0.026^{\mathrm{a}}$ \\
\hline$\leq 60$ & 82 & 46 & 36 & & & & \\
\hline \multicolumn{8}{|l|}{ Extranodal sites } \\
\hline$\geq 2$ & 39 & 30 & 9 & 4.401 & $0.036^{\mathrm{a}}$ & 0.183 & $0.036^{\mathrm{a}}$ \\
\hline$<2$ & 92 & 53 & 39 & & & & \\
\hline \multicolumn{8}{|l|}{ Clinical stage } \\
\hline III-IV & 59 & 37 & 22 & 0.019 & 0.889 & -0.012 & 0.890 \\
\hline I-II & 72 & 46 & 26 & & & & \\
\hline \multicolumn{8}{|l|}{ LDH level } \\
\hline High & 51 & 31 & 20 & 0.065 & 0.798 & -0.026 & 0.801 \\
\hline Normal & 49 & 31 & 18 & & & & \\
\hline \multicolumn{8}{|l|}{ ECOG PS } \\
\hline$\geq 2$ & 32 & 21 & 11 & 0.118 & 0.731 & 0.030 & 0.733 \\
\hline$<2$ & 98 & 61 & 37 & & & & \\
\hline \multicolumn{8}{|l|}{ IPI score } \\
\hline $3-5$ & 47 & 35 & 12 & 3.897 & $0.048^{\mathrm{a}}$ & 0.172 & $0.049^{\mathrm{a}}$ \\
\hline $0-2$ & 84 & 48 & 36 & & & & \\
\hline \multicolumn{8}{|l|}{ B symptoms } \\
\hline Yes & 29 & 20 & 9 & 0.556 & 0.456 & 0.065 & 0.460 \\
\hline No & 101 & 62 & 39 & & & & \\
\hline \multicolumn{8}{|l|}{$\mathrm{Hb}$} \\
\hline Low & 64 & 40 & 24 & 0.013 & 0.908 & -0.010 & 0.909 \\
\hline Normal & 63 & 40 & 23 & & & & \\
\hline \multicolumn{8}{|l|}{ Chemotherapy } \\
\hline $\mathrm{CHOP}+$ other & 39 & 22 & 17 & 2.006 & 0.157 & 0.160 & 0.161 \\
\hline R-CHOP & 39 & 28 & 11 & & & & \\
\hline \multicolumn{8}{|l|}{ Treatment responses } \\
\hline $\mathrm{CR}+\mathrm{PR}$ & 43 & 30 & 13 & 1.041 & 0.307 & -0.123 & 0.315 \\
\hline $\mathrm{SD}+\mathrm{PD}$ & 26 & 15 & 11 & & & & \\
\hline \multicolumn{8}{|l|}{ Subtype } \\
\hline non-GCB & 93 & 54 & 39 & 3.871 & $0.049^{\mathrm{a}}$ & -0.172 & 0.050 \\
\hline GCB & 38 & 29 & 9 & & & & \\
\hline \multicolumn{8}{|l|}{ BCL2 } \\
\hline+ & 105 & 64 & 41 & 1.320 & 0.251 & -0.100 & 0.254 \\
\hline- & 26 & 19 & 7 & & & & \\
\hline \multicolumn{8}{|l|}{ Ki67 } \\
\hline+ & 83 & 54 & 29 & 0.282 & 0.595 & 0.046 & 0.598 \\
\hline- & 48 & 29 & 19 & & & & \\
\hline
\end{tabular}

Not all cases initially included in the study are included as some patients failed to provide the relevant clinical parameters. ${ }^{a} \mathrm{P}<0.05$. BCL, B cell lymphoma; CHOP, cyclophosphamide, doxorubicin, vincristine, prednisone; CR, complete remission; DLBCL, diffuse large B cell lymphoma; ECOG PS, Eastern Cooperative Oncology Group performance status; GCB, germinal center B cell-like; Hb, hemoglobin; IPI, International Prognostic Index; LDH, lactate dehydrogenase; PD, progressive disease; PR, partial remission; R-CHOP, rituximab combined with CHOP; SD, stable disease; USP34, ubiquitin-specific-processing protease 34 . 

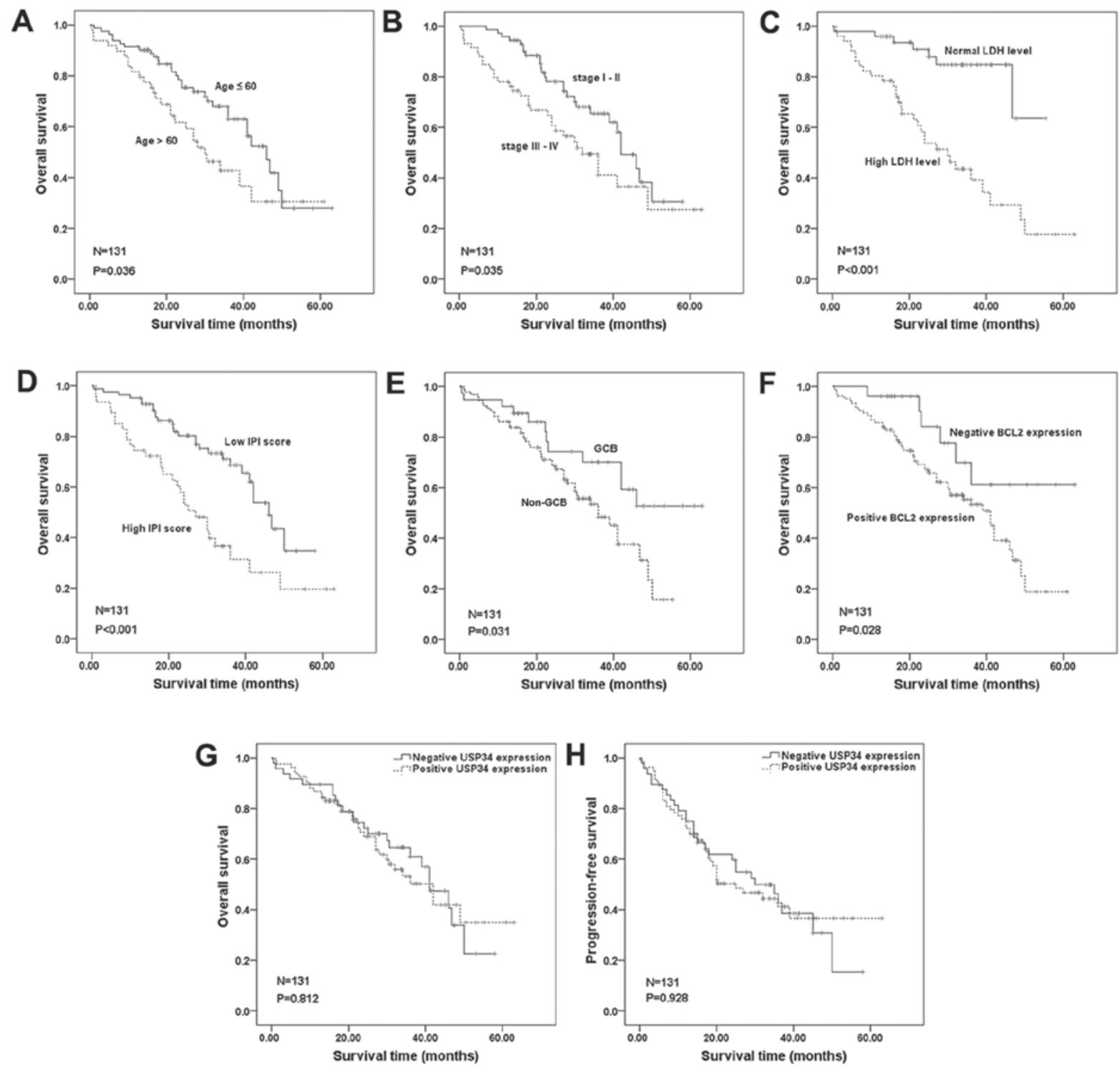

Figure 2. Univariate survival curves of clinical parameters and USP34 protein expression were analyzed for 131 patients with DLBCL. OS was determined for (A) age, (B) clinical stage, (C) LDH level, (D) IPI scores, (E) GCB subtype, (F) BCL2 expression, (G) USP34 expression and (H) progression-free survival of USP34. BCL, B cell lymphoma; DLBCL, diffuse large B-cell lymphoma; GCB, germinal center B cell-like; IPI, International Prognostic Index; LDH, lactate dehydrogenase; OS, overall survival; USP34, ubiquitin-specific-processing protease 34.

type, and poor prognosis was exhibited in the 2 p15 amplified region (21). The present study analyzed the role of USP34 in GCB-subtype DLBCL, no significant association was found between USP34 protein expression level and clinical parameters or survival. Using a TCGA dataset, a number of cases were identified with mRNA upregulation, in-frame and missense mutations of USP34. However, pathogenesis of USP34 in DLBCL, particularly in the GCB subtype, requires further investigation.

In this study, the clinical outcome of 131 patients with DLBCL was analyzed. In the univariate survival analysis, clinical stage III-IV, high LDH level, high risk IPI and non-GCB subtype were associated with poor OS of DLBCL. In addition, poor OS was seen in patients with advanced age and positive BCL2 expression. These data are consistent with previous studies, which reported that reduced OS was associated with older age, elevated LDH, advanced stage, non-GCB subtype and high risk IPI score $(3,6)$. Although USP34 protein expression was associated with older age and multiple site involvement, no significant relationship was found between USP34 level and OS, PFS or DFS. By contrast, analysis from TCGA datasets indicated that low USP34 mRNA expression level was associated with poor DFS, but not OS. The mechanisms mediating this discrepancy are uncertain, but USP34 was measured at different level (mRNA vs. protein) in the TCGA data set and in patients recruited in the present study.

Although USP34 expression was not associated with OS, increased USP34 expression in DLBCL, compared with reactive lymphoid hyperplasia, and its association with adverse clinicopathological features indicated that USP34 may serve a potential role in DLBCL pathogenesis. The potential roles of USP34 in cancer are multifocal. USP34 is closely linked to the Wnt signaling activation through the stabilization of $\beta$-catenin (14). AXIN, a key component of cellular machinery, induces phosphorylation of $\beta$-catenin. The present study 
Table II. Univariate prognostic analysis of clinicopathological parameters of 131 patients with DLBCL.

\begin{tabular}{|c|c|c|c|c|}
\hline Risk factor & $\mathrm{n}$ & 3-year OS (\%) & 5-year OS (\%) & P-value \\
\hline \multicolumn{5}{|l|}{ Sex } \\
\hline Male & 77 & 59.5 & 27.6 & \multirow[t]{2}{*}{0.185} \\
\hline Female & 54 & 47.7 & 32.5 & \\
\hline \multicolumn{5}{|l|}{ Age } \\
\hline$>60$ & 49 & 42.7 & 30.5 & \multirow[t]{2}{*}{$0.036^{\mathrm{a}}$} \\
\hline$\leq 60$ & 82 & 68.0 & 27.9 & \\
\hline \multicolumn{5}{|l|}{ Extranodal sites } \\
\hline$<2$ & 92 & 58.8 & 35.1 & \multirow[t]{2}{*}{0.160} \\
\hline$\geq 2$ & 39 & 45.9 & 15.1 & \\
\hline \multicolumn{5}{|l|}{ Clinical stage } \\
\hline III-IV & 59 & 41.2 & 27.4 & \multirow[t]{2}{*}{$0.035^{\mathrm{a}}$} \\
\hline I-II & 72 & 65.5 & 30.6 & \\
\hline \multicolumn{5}{|l|}{ LDH level } \\
\hline High & 51 & 39.2 & 17.6 & \multirow[t]{2}{*}{$<0.001^{\mathrm{a}}$} \\
\hline Normal & 49 & 84.8 & 63.6 & \\
\hline \multicolumn{5}{|l|}{ ECOG PS } \\
\hline$<2$ & 98 & 55.3 & 33.1 & \multirow[t]{2}{*}{0.526} \\
\hline$\geq 2$ & 32 & 51.9 & 26.0 & \\
\hline \multicolumn{5}{|l|}{ IPI score } \\
\hline $3-5$ & 47 & 31.4 & 19.6 & \multirow[t]{2}{*}{$<0.001^{\mathrm{a}}$} \\
\hline $0-2$ & 84 & 68.6 & 34.8 & \\
\hline \multicolumn{5}{|l|}{ B symptoms } \\
\hline Yes & 29 & 45.6 & 0.0 & \multirow[t]{2}{*}{0.171} \\
\hline No & 101 & 56.7 & 36.2 & \\
\hline \multicolumn{5}{|l|}{$\mathrm{Hb}$} \\
\hline Low & 64 & 47.4 & 8.0 & \multirow[t]{2}{*}{0.080} \\
\hline Normal & 63 & 58.1 & 48.2 & \\
\hline \multicolumn{5}{|l|}{ Subtype } \\
\hline GCB & 38 & 70.1 & 52.7 & \multirow[t]{2}{*}{$0.031^{\mathrm{a}}$} \\
\hline non-GCB & 93 & 48.4 & 15.7 & \\
\hline \multicolumn{5}{|l|}{ Treatment } \\
\hline No & 53 & 37.6 & 13.7 & \multirow[t]{2}{*}{$<0.001^{\mathrm{a}}$} \\
\hline Yes & 78 & 69.1 & 46.2 & \\
\hline \multicolumn{5}{|l|}{ Chemotherapy } \\
\hline R-CHOP & 39 & 74.0 & 74.0 & \multirow[t]{2}{*}{0.101} \\
\hline $\mathrm{CHOP}+$ other & 39 & 64.5 & 17.9 & \\
\hline USP34 & & & & \\
\hline+ & 83 & 50.2 & 34.9 & 0.812 \\
\hline- & 48 & 61.0 & 22.6 & \\
\hline BCL2 & & & & \\
\hline+ & 105 & 53.3 & 18.8 & $0.028^{\mathrm{a}}$ \\
\hline- & 26 & 61.2 & 61.2 & \\
\hline Ki67 & & & & \\
\hline+ & 83 & 57.7 & 32.5 & 0.419 \\
\hline- & 48 & 50.4 & 25.6 & \\
\hline
\end{tabular}

Not all cases initially included in the study are included as some patients failed to provide the relevant clinical parameters. ${ }^{a} \mathrm{P}<0.05$. $\mathrm{BCL}$, B cell lymphoma; CHOP, cyclophosphamide, doxorubicin, vincristine, prednisone; DLBCL, diffuse large B cell lymphoma; ECOG PS, Eastern Cooperative Oncology Group performance status; GCB, germinal center B cell-like; Hb, hemoglobin; IPI, International Prognostic Index; LDH, lactate dehydrogenase; OS, overall survival; R-CHOP, rituximab combined with CHOP; USP34, ubiquitin-specific-processing protease 34. 
Table III. Univariate prognostic analysis of clinicopathological parameters of 78 DLBCL patients' post-chemotherapy.

\begin{tabular}{llll}
\hline Risk factor & $\mathrm{n}$ & 3-year OS (\%) & 5-year OS (\%)
\end{tabular}

Sex

Male

Female

$\geq 2$

41

I-II

LDH level

High

Normal

ECOG PS

$<2$

$\geq 2$

IPI score

$\begin{array}{ll}3-5 & 30 \\ 0-2 & 48\end{array}$

B symptoms

Yes

No

$\mathrm{Hb}$

Low

Normal

Subtype

GCB

non-GCB

Chemotherapy

$\mathrm{R}-\mathrm{CHOP}$

$\mathrm{CHOP}+$ other

USP34

$+$

BCL2

$+$

$-$

Ki67

$+$

$-$

44

34

23

55

53

25

37

35

40

58

19

18

59

34

41

22

56

39

39

50

28

72.1

65.4

65.6

70.8

58.4

52.1

57.1

83.5

43.2

90.4

70.0

65.2

45.3

86.0

65.8

68.8

62.8

71.6

78.7

65.8

74.0

64.5

68.8

68.9

66.7

77.1

17

49

29

70.5

67.0
38.4

65.4

65.6

38.9

58.4

26.0

37.5

55.7

21.6

$<0.001^{\mathrm{a}}$

90.4

63.0

0.491

39.1

27.2

$0.001^{\mathrm{a}}$

57.3

0.0

0.520

55.0

16.7

0.234

71.6

78.7

$0.044^{\mathrm{a}}$

19.2

74.0

0.101

17.9

51.6

0.702

40.2

30.0

0.076

77.1

47.0

0.700

55.9

Not all cases initially included in the study are included as some patients failed to provide the relevant clinical parameters. ${ }^{\mathrm{a}} \mathrm{P}<0.05$. $\mathrm{BCL}, \mathrm{B}$ cell lymphoma; CHOP, cyclophosphamide, doxorubicin, vincristine, prednisone; DLBCL, diffuse large B cell lymphoma; ECOG PS, Eastern Cooperative Oncology Group performance status; GCB, germinal center B cell-like; Hb, hemoglobin; IPI, International Prognostic Index; LDH, lactate dehydrogenase; OS, overall survival; R-CHOP, rituximab combined with CHOP; USP34, ubiquitin-specific-processing protease 34. 
A

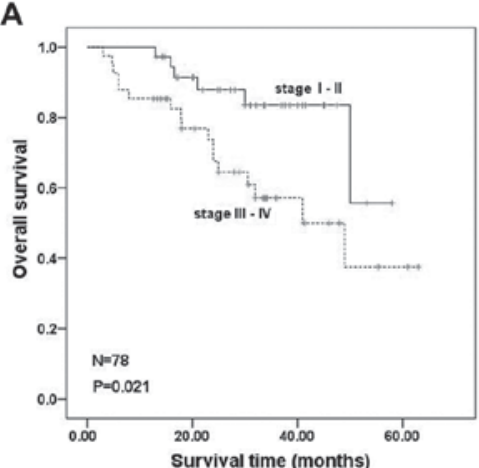

D

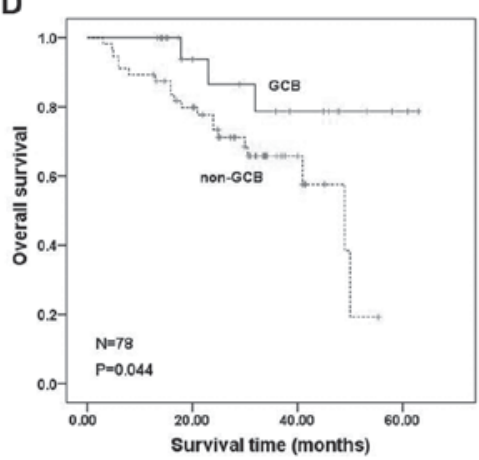

B

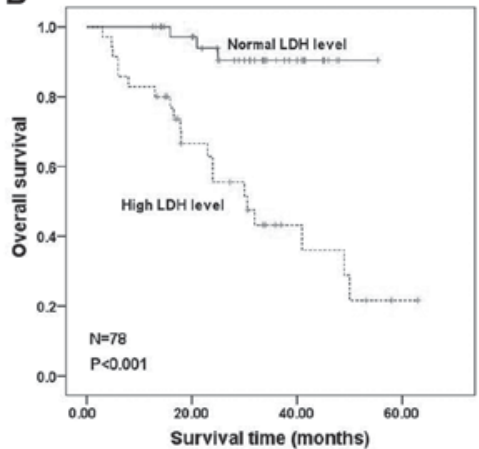

E

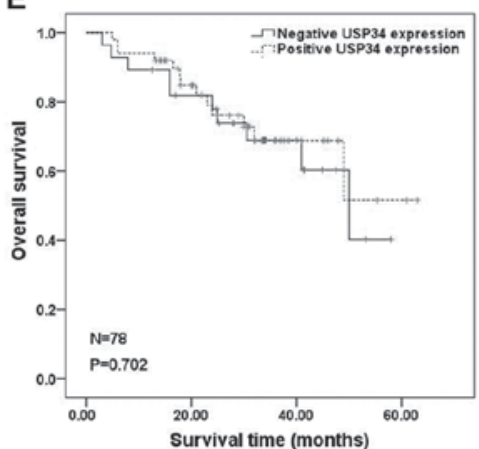

C

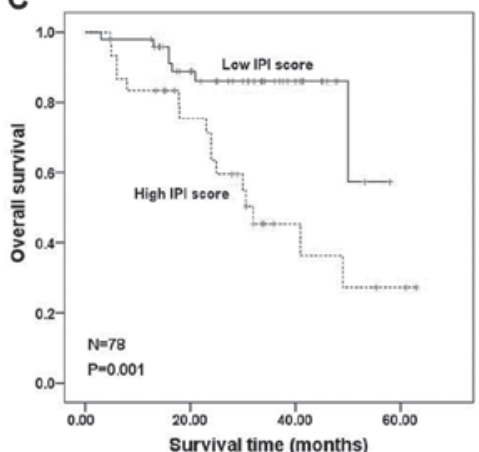

$\mathbf{F}$

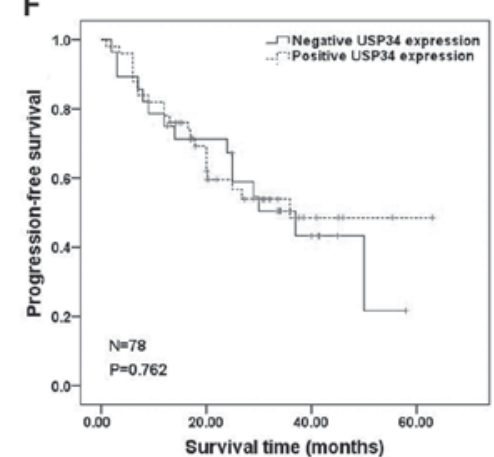

Figure 3. Univariate survival curves of clinical parameters and USP34 protein expression were analyzed for 78 patients with DLBCL that received chemotherapy. OS was determined for (A) of clinical stage, (B) LDH level, (C) IPI score, (D) GCB subtype (E) USP34 expression, and (F) progression-free survival of USP34. DLBCL, diffuse large B-cell lymphoma; IPI, International Prognostic Index; LDH, lactate dehydrogenase; OS, overall survival; USP34, ubiquitin-specific-processing protease 34 .

Altered in $15(31 \%)$ of 48 cases/patients

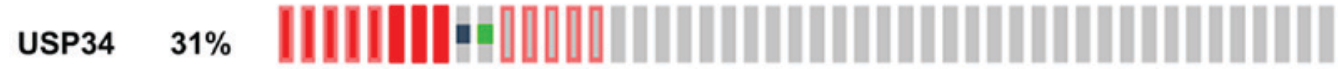

Genetic alteration

Amplification \mRNA upregulation

Missense mutation (putative passenger)

Figure 4. Representation of the USP34 genetic alterations, including amplification, mRNA upregulation, in-frame mutation and missense mutation identified in 48 DLBCL data sets (TCGA, provisional) that were downloaded from cBioPortal. DLBCL, diffuse large B-cell lymphoma; TCGA, The Cancer Genome Atlas; USP34, ubiquitin-specific-processing protease 34.
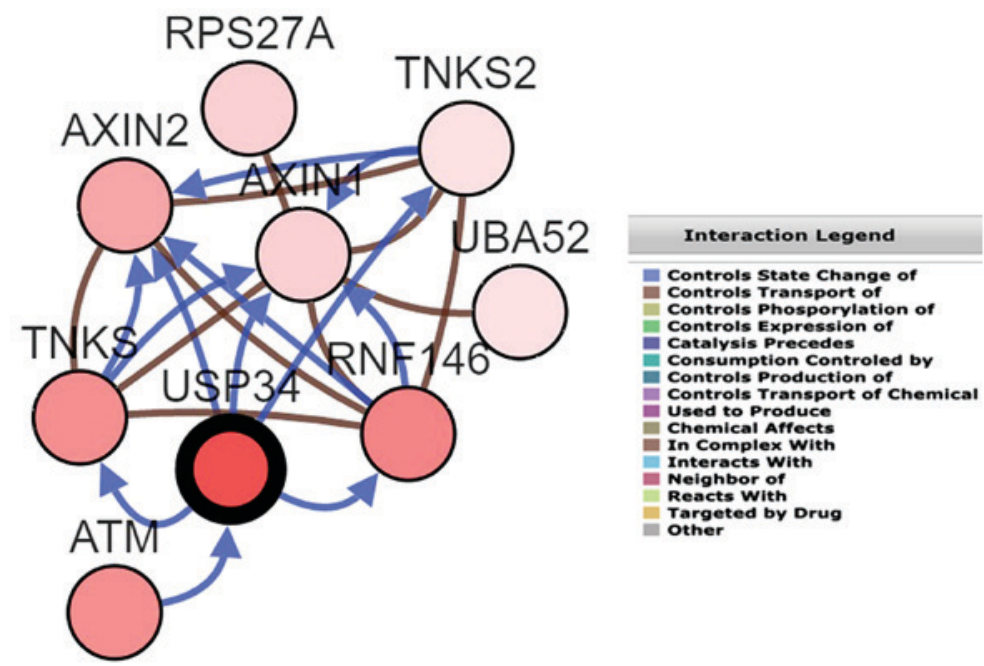

Figure 5. Diagram of the gene network related to USP34 in DLBCL were downloaded from cBioPortal and circles indicated genes. ATM, ataxia telangiectasia-mutated; $D L B C L$, diffuse large B-cell lymphoma; $R N F 146$, ring-finger 146; RPS27A, ribosomal protein S27a; TNKS, tankyrase; UBA52, ubiquitin A-52 residue ribosomal protein fusion product 1; USP34, ubiquitin-specific-processing protease 34. 

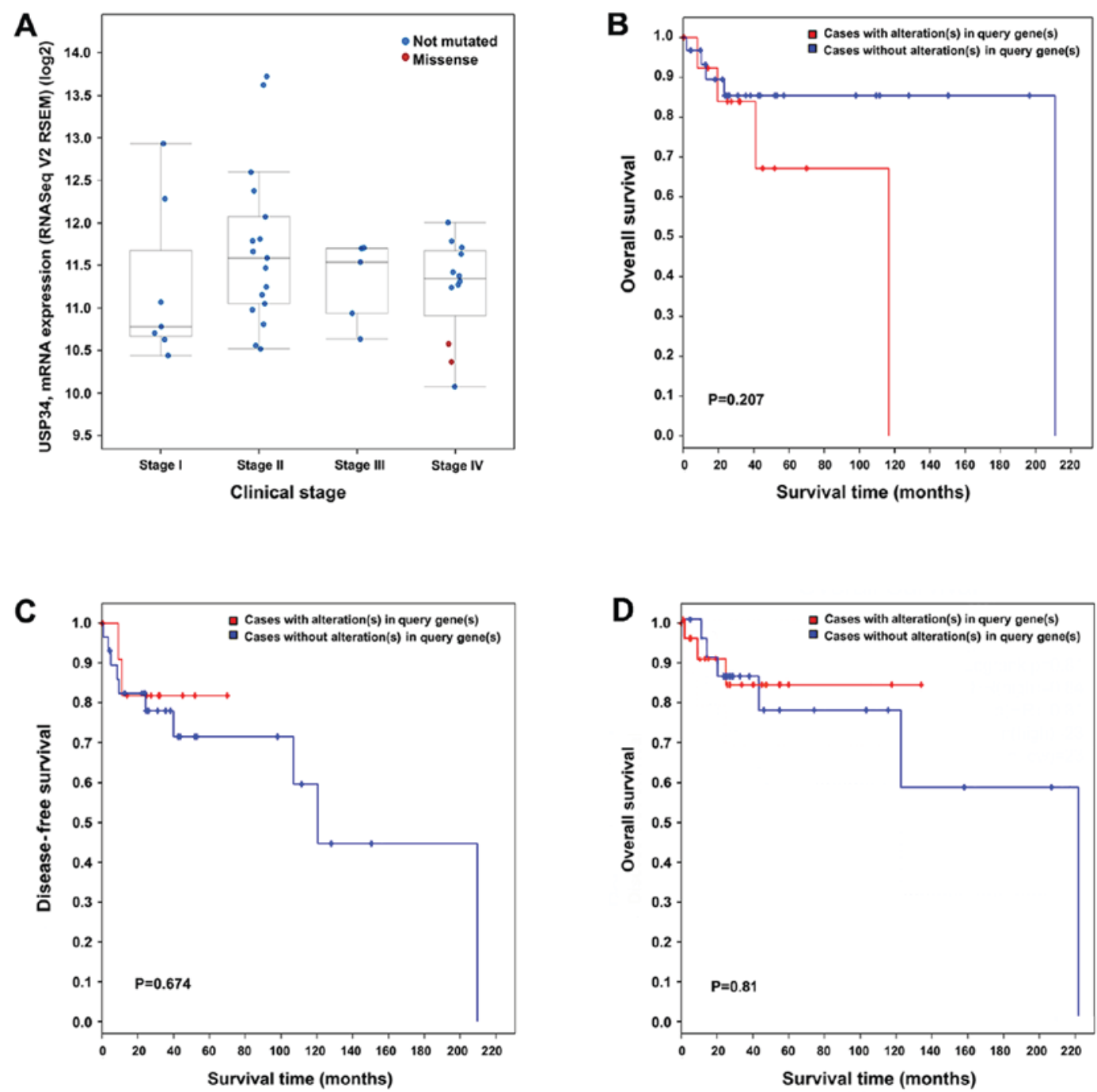

Figure 6. Clinical parameter and survival diagrams of USP34 in 48 DLBCL data sets (TCGA, provisional) downloaded from cBioPortal. (A) No association of the USP34 mRNA level with clinical stage of DLBCL (P>0.05). (B) Overall survival of the USP34 alterations in patients with DLBCL. (C) Disease free survival rate of patients with DLBCL and USP34 alterations in TCGA dataset: 2 cases relapsed of the 13 cases with alterations, whereas 10 cases relapsed among the 30 cases without alterations and the median time of disease free survival was 120.53 months. (D) Overall survival of the USP34 alterations in patients with DLBCL in TCGA dataset. DLBCL, diffuse large B-cell lymphoma; TCGA, The Cancer Genome Atlas; USP34, ubiquitin-specific-processing protease 34 .
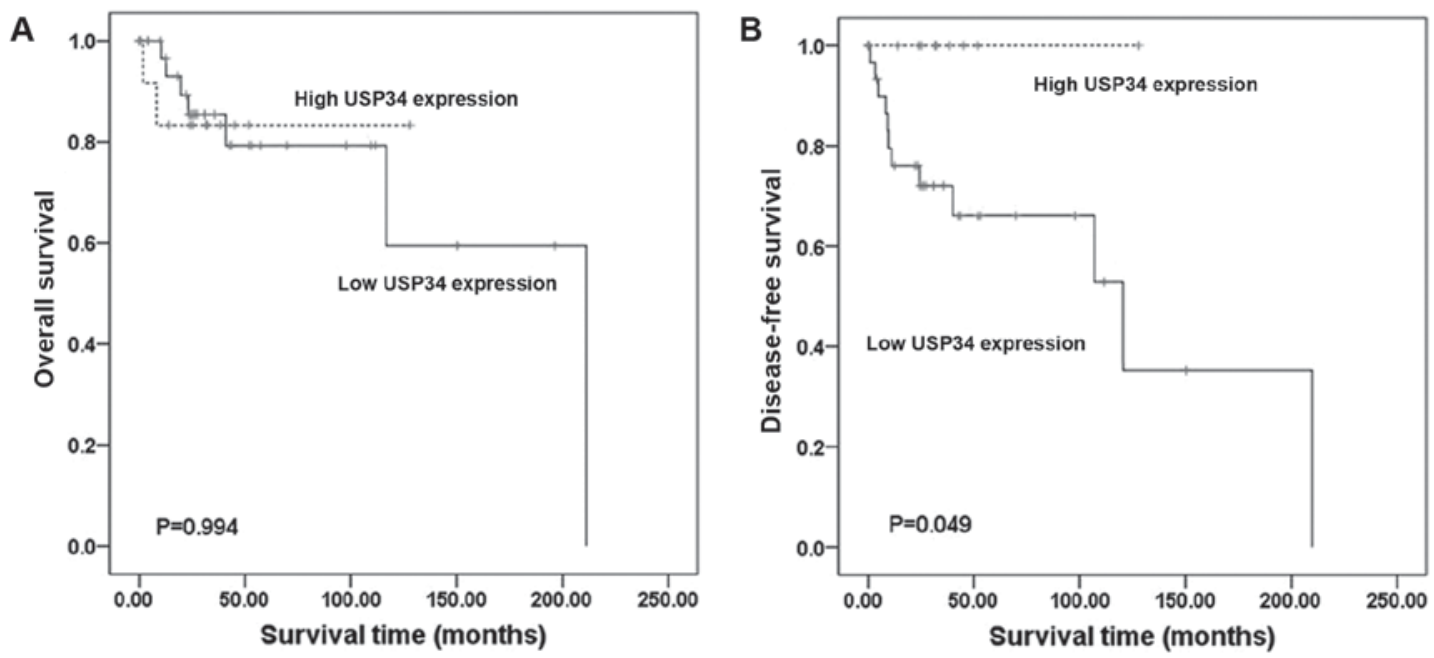

Figure 7. Univariate survival curves of USP34 mRNA expression were analyzed for patients with DLBCL in TCGA datasets (TCGA, provisional). (A) Overall survival of USP34 expression level. (B) Disease free survival of USP34 expression level. DLBCL, diffuse large B-cell lymphoma; TCGA, The Cancer Genome Atlas; USP34, ubiquitin-specific-processing protease 34. 
identified a potential network connection of USP34 with AXIN1 and AXIN2 in TCGA, but this needs to be verified. In DLBCL, potentiated Wnt/ $\beta$-catenin signal transduction was previously reported, along with forkhead box P1 overexpression, and Wnt-targeted therapy was examined in DLBCL (38). In addition, USP34 was reported to be a DNA damage-responsive protein and regarded as the downstream target of ataxia telangiectasia-mutated in DNA damage (9), and the interaction between them was identified in TCGA. A link between DSP34 and the NF- $\kappa \mathrm{B}$ pathway is also described (39). NF- $\kappa \mathrm{B}$ and $\mathrm{B}$ cell-receptor signaling may be activated by the E3 ubiquitin ligases cellular inhibitor of apoptosis 1 and 2 in activated B cell-like DLBCL. USP34 was reported to be distributed in cytosol and to negatively regulate $\mathrm{NF}-\kappa \mathrm{B}$ signaling pathway via the downstream CARMA1/BCL10/MALT1-inhibitor of NF- $\kappa \mathrm{B}$ kinase complex in Tlymphocytes (16). In addition, USP34 was reported to prevent Toll pathway and immune deficiency-dependent immune signal in Drosophila (15). In a recent study, E3 ubiquitin-protein ligase pellino 1, which serves a pivotal role in the activation of $N F-\kappa B$ pathway and Toll-like receptor signaling, was demonstrated to be associated with a poor prognosis of patients with DLBCL (40). The research of USP34 in DLBCL is limited, and future studies to explore its function are needed.

In conclusion, high USP34 protein expression was identified in patients with DLBCL, and was associated with older age, GCB subtype, multiple extranodal extension and high risk IPI. Future studies will focus on exploring the role of USP34 in the pathogenesis of DLBCL.

\section{Acknowledgements}

Not applicable.

\section{Funding}

The present study was funded by The Key Programs of University Scientific Research of Guangxi Education Agency (grant no. ZD2014033), The Key Programs of Guangxi Natural Science Fund (grant no. 2015GXNSFDA139028) and The Basic Ability Improvement Project for Young and Middle-aged Teachers in Guangxi Universities (grant no. 2017KY0091).

\section{Availability of data and materials}

The datasets used and/or analyzed during the current study are available from the corresponding authors on reasonable request.

\section{Authors' contributions}

CL and LH collected data from clinical and public dataset, performed statistical analysis and drafted the manuscript. CL, HL and QZ carried out the clinical samples gathering and in-house immunoreactivity. GC and WW participated in the design of the study and language modification. YG and $\mathrm{ZP}$ evaluated the clinical dataset, morphology and immunoreactivity. ZF conceived of the study and helped to edit the manuscript. All authors read and approved the final manuscript.

\section{Ethics approval and consent to participate}

This study was approved by the Ethical Committee of the First Affiliated Hospital of Guangxi Medical University. Written informed consents were signed by all the patients involved to ensure their approval of the data used in this research.

\section{Patient consent for publication}

All patients signed to consent for publishing their individual clinical data.

\section{Competing interests}

The authors declare that they have no competing interests.

\section{References}

1. Siegel RL, Miller KD and Jemal A: Cancer statistics, 2016. CA Cancer J Clin 66: 7-30, 2016.

2. Swerdlow SH, Campo E, Pileri SA, Harris NL, Stein H, Siebert R, Advani R, Ghielmini M, Salles GA, Zelenetz AD and Jaffe ES: The 2016 revision of the World Health Organization classification of lymphoid neoplasms. Blood 127: 2375-2390, 2016.

3. Seo S, Hong JY, Yoon S, Yoo C, Park JH, Lee JB, Park CS, Huh J, Lee Y, Kim KW, et al: Prognostic significance of serum beta-2 microglobulin in patients with diffuse large B-cell lymphoma in the rituximab era. Oncotarget 7: 76934-76943, 2016.

4. Nowakowski GS and Vitolo U: Recent advances in clinical studies and the evolving role of subtyping for patients with diffuse large B-cell lymphoma. Future Oncol 13: 859-862, 2017.

5. Oki Y, Ewer MS, Lenihan DJ, Fisch MJ, Hagemeister FB, Fanale M, Romaguera J, Pro B, Fowler N, Younes A, et al: Pegylated liposomal doxorubicin replacing conventional doxorubicin in standard R-CHOP chemotherapy for elderly patients with diffuse large B-cell lymphoma: An open label, single arm, phase II trial. Clin Lymphoma Myeloma Leuk 15: 152-158, 2015.

6. Friedberg JW: Relapsed/refractory diffuse large B-cell lymphoma. Hematol Am Soc Hematol Educ Program 2011: 498-505, 2011.

7. Zhu L, Yang S, He S, Qiang F, Cai J, Liu R, Gu C, Guo Z, Wang C, Zhang W, et al: Downregulation of ubiquitin-specific protease 14 (USP14) inhibits breast cancer cell proliferation and metastasis, but promotes apoptosis. J Mol Histol 47: 69-80, 2016.

8. Piao S, Liu Y, Hu J, Guo F, Ma J, Sun Y and Zhang B: USP22 is useful as a novel molecular marker for predicting disease progression and patient prognosis of oral squamous cell carcinoma. PLoS One 7: e42540, 2012.

9. Sy SM, Jiang J, O WS, Deng Y and Huen MS: The ubiquitin specific protease USP34 promotes ubiquitin signaling at DNA double-strand breaks. Nucleic Acids Res 41: 8572-8580, 2013.

10. Phillips AH, Zhang Y, Cunningham CN, Zhou L, Forrest WF, Liu PS, Steffek M, Lee J, Tam C, Helgason E, et al: Conformational dynamics control ubiquitin-deubiquitinase interactions and influence in vivo signaling. Proc Natl Acad Sci USA 110: 11379-11384, 2013.

11. Zeng Z, Wu HX, Zhan N, Huang YB, Wang ZS, Yang GF, Wang P and Fu GH: Prognostic significance of USP10 as a tumor-associated marker in gastric carcinoma. Tumour Biol 35: 3845-3853, 2014.

12. Wang Y, Wang J, Zhong J, Deng Y, Xi Q, He S, Yang S, Jiang L, Huang M, Tang C and Liu R: Ubiquitin-specific protease 14 (USP14) regulates cellular proliferation and apoptosis in epithelial ovarian cancer. Med Oncol 32: 379, 2015.

13. Zhang Y, Yao L, Zhang X, Ji H, Wang L, Sun S and Pang D: Elevated expression of USP22 in correlation with poor prognosis in patients with invasive breast cancer. J Cancer Res Clin Oncol 137: 1245-1253, 2011.

14. Lui TT, Lacroix C, Ahmed SM, Goldenberg SJ, Leach CA, Daulat AM and Angers S: The ubiquitin-specific protease USP34 regulates axin stability and Wnt/ $\beta$-catenin signaling. Mol Cell Biol 31: 2053-2065, 2011. 
15. Engel E, Viargues P, Mortier M, Taillebourg E, Couté Y, Thevenon D and Fauvarque MO: Identifying USPs regulating immune signals in Drosophila: USP2 deubiquitinates Imd and promotes its degradation by interacting with the proteasome. Cell Commun Signal 12: 41, 2014.

16. Poalas K, Hatchi EM, Cordeiro N, Dubois SM, Leclair HM, Leveau C, Alexia C, Gavard J, Vazquez A and Bidère N: Negative regulation of NF- $\kappa \mathrm{B}$ signaling in $\mathrm{T}$ lymphocytes by the ubiquitin-specific protease USP34. Cell Commun Signal 11: 25, 2013.

17. Zhao S, Tian Y, Zhang W, Xing X, Li T, Liu H, Huang T, Ning Y, Zhao $\mathrm{H}$ and Chen ZJ: An association study between USP34 and polycystic ovary syndrome. J Ovarian Res 8: 30, 2015.

18. Mimouni-Bloch A, Yeshaya J, Kahana S, Maya I and Basel-Vanagaite L: A de-novo interstitial microduplication involving 2p16.1-p15 and mirroring 2p16.1-p15 microdeletion syndrome: Clinical and molecular analysis. Eur J Paediatr Neurol 19: 711-715, 2015.

19. Kwiecinska A, Ichimura K, Berglund M, Dinets A, Sulaiman L, Collins VP, Larsson C, Porwit A and Lagercrantz SB Amplification of $2 p$ as a genomic marker for transformation in lymphoma. Genes Chromosomes Cancer 53: 750-768, 2014.

20. Scholtysik R, Kreuz M, Hummel M, Rosolowski M, Szczepanowski M, Klapper W, Loeffler M, Trümper L, Siebert R and Küppers R; Molecular Mechanisms in Malignant Lymphomas Network Project of the Deutsche Krebshilfe: Characterization of genomic imbalances in diffuse large B-cell lymphoma by detailed SNP-chip analysis. Int J Cancer 136: 1033-1042, 2015.

21. Taskinen M, Louhimo R, Koivula S, Chen P, Rantanen V, Holte H, Delabie J, Karjalainen-Lindsberg ML, Björkholm M, Fluge $\varnothing$, et al: Deregulation of COMMD1 is associated with poor prognosis in diffuse large B-cell lymphoma. PLoS One 9: e91031, 2014.

22. Trifonov V, Pasqualucci L, Dalla Favera R and Rabadan R: MutComFocal: An integrative approach to identifying recurrent and focal genomic alterations in tumor samples. BMC Syst Biol 7: 25, 2013 .

23. Mo CH, Gao L, Zhu XF, Wei KL, Zeng JJ, Chen G and Feng ZB: The clinicopathological significance of UBE2C in breast cancer: A study based on immunohistochemistry, microarray and RNA-sequencing data. Cancer Cell Int 17: 83, 2017.

24. Boustani MR, Khoshnood RJ, Nikpasand F, Taleshi Z, Ahmadi K, Yahaghi E and Goudarzi PK: Overexpression of ubiquitin-specific protease 2a (USP2a) and nuclear factor erythroid 2-related factor 2 (Nrf2) in human gliomas. J Neurol Sci 363: 249-252, 2016.

25. Wang Y, Liu Y, Yang B, Cao H, Yang CX, Ouyang W, Zhang SM, Yang GF, Zhou FX, Zhou YF and Xie CH: Elevated expression of USP9X correlates with poor prognosis in human non-small cell lung cancer. J Thorac Dis 7: 672-679, 2015.

26. Wang Z, Zhu L, Guo T, Wang Y and Yang J: Decreased H2B monoubiquitination and overexpression of ubiquitin-specific protease enzyme 22 in malignant colon carcinoma. Hum Pathol 46: 1006-1014, 2015

27. Tang B, Tang F, Li B, Yuan S, Xu Q, Tomlinson S, Jin J, Hu W and He S: High USP22 expression indicates poor prognosis in hepatocellular carcinoma. Oncotarget 6: 12654-12667, 2015.
28. Ning Z, Wang A, Liang J, Xie Y, Liu J, Feng L, Yan Q and Wang Z: USP22 promotes the G1/S phase transition by upregulating FoxM1 expression via $\beta$-catenin nuclear localization and is associated with poor prognosis in stage II pancreatic ductal adenocarcinoma. Int J Oncol 45: 1594-1608, 2014.

29. Ao N, Liu Y, Bian X, Feng H and Liu Y: Ubiquitin-specific peptidase 22 inhibits colon cancer cell invasion by suppressing the signal transducer and activator of transcription 3/matrix metalloproteinase 9 pathway. Mol Med Rep 12: 2107-2113, 2015.

30. Zhang L, Xu B, Qiang Y, Huang H, Wang C, Li D and Qian J: Overexpression of deubiquitinating enzyme USP28 promoted non-small cell lung cancer growth. J Cell Mol Med 19: 799-805, 2015.

31. Zhao Y, Zhang B, Lei Y, Sun J, Zhang Y, Yang S and Zhang X: Knockdown of USP39 induces cell cycle arrest and apoptosis in melanoma. Tumour Biol 37: 13167-13176, 2016.

32. Colomo L, Lopez-Guillermo A, Perales M, Rives S, Martínez A, Bosch F, Colomer D, Falini B, Montserrat E and Campo E: Clinical impact of the differentiation profile assessed by immunophenotyping in patients with diffuse large B-cell lymphoma. Blood 101: 78-84, 2003.

33. Liao W: Different expression status of apoptosis related proteins Fas, FasL, Bcl-2 and survivin in GCB and non-GCB immuno-subtypes of diffuse large B-cell lymphoma in Guangxi, China (unpublished PhD thesis). Guangxi Medical University, 2012.

34. Gan TQ, Xie ZC, Tang RX, Zhang TT, Li DY, Li ZY and Chen G: Clinical value of miR-145-5p in NSCLC and potential molecular mechanism exploration: A retrospective study based on GEO, qRT-PCR and TCGA data. Tumour Biol 39: 1010428317691683 2017.

35. Zhang X, Ye ZH, Liang HW, Ren FH, Li P, Dang YW and Chen G: Down-regulation of miR-146a-5p and its potential targets in hepatocellular carcinoma validated by a TCGA- and GEO-based study. FEBS Open Bio 7: 504-521, 2017.

36. Wei DM, Chen ZX, He RQ, Shi L, Zhou S, Li W, Chen G, PengZ, Dang Y and Luo D: Genomic alterations andprotein expression of STAT4 in pancreatic cancer: A study of bioinformatics based on public data and immunohistochemistry validation with 241 tissue samples. Int J Clin Exp Pathol 9: 9761-9774, 2016.

37. Kubuschok B, Held G and Pfreundschuh M: Management of diffuse large B-cell lymphoma (DLBCL). Cancer Treat Res 165: 271-288, 2015 .

38. Walker MP, Stopford CM, Cederlund M, Fang F, Jahn C, Rabinowitz AD, Goldfarb D, Graham DM, Yan F, Deal AM, et al: FOXP1 potentiates Wnt/ $\beta$-catenin signaling in diffuse large B cell lymphoma. Sci Signal 8: ra12, 2015.

39. Yang Y, Kelly P, Shaffer AL III, Schmitz R, Yoo HM, Liu X, Huang DW, Webster D, Young RM, Nakagawa M, et al: Targeting non-proteolytic protein ubiquitination for the treatment of diffuse large B cell lymphoma. Cancer Cell 29: 494-507, 2016.

40. Choe JY, Park M, Yun JY, Na HY, Go H, Kim HJ, Oh S and Kim JE: PELI1 expression is correlated with MYC and BCL6 expression and associated with poor prognosis in diffuse large B-cell lymphoma. Mod Pathol 29: 1313-1323, 2016. 